\title{
Hate Speech - Ein Thema für die Linguistik
}

\author{
Konstanze Marx
}

\section{Abstract}

Linguistic expressions used in order to discriminate people because of their affiliation to a certain group can be summarized as Hate Speech. Hate Speech is often found in Social Media. The topic is rather complex and ought to be investigated interdisciplinarily. This paper delivers an excerpt of the linguistic point of view. It shows how Hate Speech can be distinguished from insulting or criticizing. It also shows that there are two parallel discourses about Hate Speech, one rather populist right wing oriented and one rather reflecting the subject as verbal violence. By means of two elaborated examples, the concrete use and dynamics of Hate Speech are analyzed.

\section{Einleitung}

Der Linguist Jörg Meibauer hat im Jahr 2013 einen Online-Sammelband zum Thema »Hate Speech" (Hassrede) herausgegeben, in dem er - und das ist symptomatisch für die wissenschaftliche Auseinandersetzung mit diesem Phänomen - »interdisziplinäre Beitrăge« zur aktuellen Diskussion zusammengestellt hat. Wie die Beiträge von Mathias Hong ${ }^{1}$, Christian Frerix $^{2}$ oder Janina Lehmann ${ }^{3}$ zeigen, handelt es sich zweifelsohne um einen juristischen Gegenstand. Fragen zur Regulierung, zu rechtlichen Konsequenzen und zu möglichen Ansprüchen drängen sich im Zusammenhang mit Hate Speech geradezu auf. Auch aus kriminologischer Perspektive erscheint es lohnenswert danach zu fragen, welche Auswirkungen Straftaten

$1 \quad$ Hong, Hate Speech im Internet - Grundrechtliche Rahmenbedingungen ihrer Regulierung, in diesem Band, S. $59 \mathrm{ff}$.

2 Frerix, Arbeitnehmerăußerungen in sozialen Netzwerken als Klundigungsgrund, in diesem Band, S. $127 \mathrm{ff}$.

3 Lehmann, Hate Speech: Rechtsansprüche und Rechtsprechung, in diesem Band, S. $89 \mathrm{ff}$. 
im Netz auf das individuelle Verstăndnis von Normen für den physischen Raum haben oder wie die Strafverfolgung im Netz gesichert werden kann. ${ }^{4}$ Der soziologische Blick würde sich auf Hate Speech als spezifisches menschliches Verhalten in sozialen und gesellschaftlichen Räumen richten, der historische fokussierte z. B. Judenhass als prototypisches Beispiel für Hate Speech. ${ }^{5}$ Die Literaturwissenschaft thematisiert Hate Speech als literarische Rhetorik ${ }^{6}$, für die Religionswissenschaft kann Hassrede untersuchenswert sein, weil sie insbesondere in religiös motivierten Diskursen vorkommt (vgl. "Glaubenskrieger «). ${ }^{7}$ Ein philosophischer Zugang könnte die ethische Kategorisierung der Hate Speech-Handlungen sein, psychologisch interessant ist die Definition der Emotion Hass einerseits und der Abgleich mit dem, was durch Hate Speech ausgedrückt wird, andererseits. Diese Liste ließe sich noch fortführen. Für den vorliegenden Aufsatz ist jedoch insbesondere relevant, dass Hate Speech eine sprachliche Handlung darstellt und damit eine Untersuchung aus linguistischer Perspektive motiviert.

Hassrede, so umschreibt es Meibauer, ist der ssprachliche[n] Ausdruck von Hass gegen Personen oder Gruppen [...], insbesondere durch die Verwendung von Ausdrücken, die der Herabsetzung und Verunglimpfung von Bevölkerungsgruppen dienen ${ }^{8}{ }^{8}$ Diese Definition knüpft unmittelbar an die politische Definition an. Unter diesen Begriff fallen danach wall forms of expression which spread, incite, promote or justify racial hatred, xenophobia, anti-Semitism or other forms of hatred based on intolerance, including: intolerance expressed by aggressive nationalism and ethnocentrism, discrimination and hostility against minorities, migrants and people

4 Rüdiger, \#NoHate. München \& Hatespeech in Onlinegames. Wir müssen mal reden!, 2016, https://www.linkedin.com/pulse/munchen-hatespeech-online games-wir-müssen-mal-reden-rüdiger, abgerufen am 25.7.2017.

5 Friesel, Juden-Hass gestem und heute: Ein historischer Blick auf 130 Jahre judeophobische Feindseligkeit, in: Meibauer (Hrsg.), Hassrede/Hatespeech. Interdisziplinăre Beitrăge zu einer aktuellen Diskussion, 2013, S. 17-27.

6 Meyer-Sickendiek, Hate Speech als literarische Rhetorik, oder: Wie man mit Judith Butler sarkastische Texte lesen kann, in: Meibauer (Hrsg.), Hassrede/Hatespeech. Interdisziplinäre Beiträge zu einer aktuellen Diskussion, 2013, S. 95-120.

7 Brodnig, Hass im Netz. Was wir gegen Hetze, Mobbing und Lugen tun kơnnen, 2016.

8 Meibauer, Hassrede - von der Sprache zur Politik, in: Meibauer (Hrsg.), Hassrede/Hate Speech. Interdisziplinăre Beitrăge zu einer aktuellen Diskussion, 2013, S. 1 . 
of immigrant origin . $^{9}$ Damit ist Hate Speech zum Ausdruck gebrachte Diskriminierung, also sprachliches Zeugnis von »Ungleichbehandlung auf kategorialer Basis ${ }^{10}{ }^{10}$ Als juristischen Bezugspunkt nennt u. a. Stefanowitsch" $^{11}$ den Tatbestand der Volksverhetzung. Im $\S 130$ des Strafgesetzbuches heißt es dazu:

(1) Wer in einer Weise, die geeignet ist, den öffentlichen Frieden zu stören, 1. gegen eine nationale, rassische, religiose oder durch ihre ethnische Herkunft bestimmte Gruppe, gegen Teile der Bevoblkerung oder gegen einen Einzelnen wegen seiner Zugehörigkeit zu einer vorbezeichneten Gruppe oder zu einem Teil der Bevơlkerung zum Hass aufstachelt, zu Gewalt- oder Willkürmaßnahmen auffordert oder 2. die Menschenwürde anderer dadurch angreift, dass er eine vorbezeichnete Gruppe, Teile der Bevőlkerung oder einen Einzelnen wegen seiner Zugehörigkeit zu einer vorbezeichneten Gruppe oder zu einem Teil der Bevolkerung beschimpft, bőswillig verăchtlich macht oder verleumdet, wird mit Freiheitsstrafe von drei Monaten bis zu fünf Jahren bestraft.

(2) Mit Freiheitsstrafe bis zu drei Jahren oder mit Geldstrafe wird bestraft, wer 1. eine Schrift (\$11 Absatz 3) verbreitet oder der Öffentlichkeit zugänglich macht oder einer Person unter achtzehn Jahren eine Schrift ( $(11$ Absatz 3) anbietet, uberlasst oder zugänglich macht, die a) zum Hass gegen eine in Absatz 1 Nummer 1 bezeichnete Gruppe, gegen Teile der Bevolkerung oder gegen einen Einzelnen wegen seiner Zugehörigkeit zu einer in Absatz 1 Nummer 1 bezeichneten Gruppe oder zu einem Teil der Bevollkerung aufstachelt, b) zu Gewalt- oder Willkurmaßnahmen gegen in Buchstabe a genannte Personen oder Personenmehrheiten auffordert oder c) die Menschenwirde von in Buchstabe a genannten Personen oder Personenmehrheiten dadurch angreift, dass diese beschimptt, bőswillig verächtlich gemacht oder verleumdet werden, 2. einen in Nummer 1 Buchstabe a bis $\mathrm{c}$ bezeichneten Inhalt mittels Rundfunk oder Telemedien einer Person unter achtzehn Jahren oder der Öffentlichkeit zugänglich macht oder 3. eine Schrift (§ II Absatz 3) des in Nummer 1 Buchstabe a bis $\mathbf{c}$ bezeichneten Inhalts herstellt, bezieht, liefert, vorrátig hallt, anbietet, bewirbt oder es unternimmt, diese Schrift ein- oder auszufuhren, um sie oder aus ihr gewonnene Stacke im Sinne der Nummer 1 oder Nummer 2 zu verwenden oder einer anderen Person eine solche Verwendung zu ermoglichen.

9 Empfehlung Nr. R (97) 20 des Kommittees der Minister des Europarates über whate speech «, angenommen am 30. Oktober 1997.

10 Graumann/Wintermantel, Diskriminierende Sprechakte. Ein funktionaler Ansatz, in: Herrmann/Krămer/Kuch (Hrsg.), Verletzende Worte. Die Grammatik sprachlicher Missachtung, 2007, S. 147-178. Vgl. auch Graumann, Verbal discrimination: a new chapter in the social psychology of aggression, Journal for the Theory of Social Behavior 1998, $41 \mathrm{ff}$.

11 Stefanowitsch, Was ist Uberhaupt Hate Speech, 2016, https://www.amadeuantonio-stiftung.de/hatespeech/was-ist-ueberhaupt-hate-speech/, abgerufen am 25.7.17. 
(3) Mit Freiheitsstrafe bis zu funf Jahren oder mit Geldstrafe wird bestraft, wer eine unter der Herrschaft des Nationalsozialismus begangene Handlung der in $\S 6$ Abs. 1 des Völkerstrafgesetzbuches bezeichneten Art in einer Weise, die geeignet ist, den öffentlichen Frieden zu storen, offentlich oder in einer Versammlung billigt, leugnet oder verharmlost.

(4) Mit Freiheitsstrafe bis zu drei Jahren oder mit Geldstrafe wird bestraft, wer offentlich oder in einer Versammlung den offentlichen Frieden in einer die Würde der Opfer verletzenden Weise dadurch stört, dass er die nationalsozialistische Gewalt- und Willkurherrschaft billigt, verherrlicht oder rechtfertigt. [...]

Bei dem Versuch, diese Aspekte zusammenzuführen, kommen Fragen auf. Kann man folglich davon ausgehen, dass jede zum Ausdruck gebrachte Diskriminierung Hate Speech ist? Oder anders gefragt: Gibt es uberhaupt Diskriminierung, die nicht Hate Speech ist? In welchen Zusammenhang kőnnen Diskriminierung und Hate Speech gebracht werden, wenn die im Volksverhetzungsparagraphen genannte politische Gesinnung berucksichtigt wird? Wie verhalt es sich beispielsweise mit Diskriminierung auf Basis des Geschlechts oder der sexuellen Orientierung? Die Fragen deuten schon an, dass es sich hier um ein recht unübersichtliches Phänomen handelt. Die Fragen, die sich aus sprachwissenschaftlicher Sicht stellen, beziehen sich in diesem Zusammenhang auch darauf, ob es sprachliche Indikatoren gibt, die einen Unterschied zwischen Diskriminierung und Hate Speech anzeigen. Übergeordnet wäre die Frage, welche Formen sprachlicher Umsetzung es uberhaupt furr diese Phänomene gibt. Eng damit in Verbindung steht das Gewalt- und Machtpotenzial von Sprache. Eine năchste wichtige Frage aus linguistischer Sicht ist, wie das Phănomen im Diskurs be- und verhandelt wird. Das lässt sich besonders gut in den Sozialen Medien nachvollziehen. Entsprechend bilden Twitter und Facebook die Quelle fur die im vorliegenden Beitrag zitierten Belege.

\section{Parallele Hate Speech-Diskurse}

Hate Speech ist nicht nur ausagierte verbale Gewalt, sondern auch ein Gegenstand, uber den ein Diskurs gefuhrt wird. Dieser war in letzter Zeit immer besonders dann sehr intensiv, wenn in den Nachrichten uber das Netzwerkdurchsetzungsgesetz (NetzDG) berichtet wurde. Das NetzDG wurde am 30. Juni 2017 im Bundestag mit dem Ziel verabschiedet, Hasskriminalităt und Fake News einzudämmen. Verschiedene Faktoren haben dazu beigetragen, dass diese Thematik in den letzten beiden Jahren einem breiteren Publikum zugänglich wurde. Dazu gehören der Geflüchtetendiskurs, der amerikanische Wahlkampf, Videobotschaften von Politikerinnen, 
die Anfeindungen ausgesetzt waren und sind (z. B. Katrin Göring-Eckardt oder Claudia Roth), oder die öffentlichen Reaktionen von Journalist_innen auf Hasskommentare und -briefe (z. B. im Rahmen von Hate PoetrySlams). Auch mit den wahrnehmbaren Überlegungen zum Gesetzesentwurf (etwa zur Gründung der Arbeitsgruppe zum Umgang mit strafbaren Inhalten in sozialen Netzwerken im Bundesministerium der Justiz und für Verbraucherschutz im Jahr 2015, der Vorstellung des Gesetzesentwurfs durch Heiko Maas im Frtihjahr 2017, der Abstimmung im Bundestag im Juni 2017) gingen jeweils Kommentare in den Sozialen Netzwerken einher.

Der Eindruck großer Konfusion entsteht nun, weil es Paralleldiskurse zu Hate Speech gibt. Es lassen sich grob zwei Perspektiven auf vergleichbares sprachliches Material erkennen. So wird die Hate SpeechEtikettierung erstens als Instrument dafür begriffen, Meinungsăußerungen als Rechtsverstoß zu deklarieren. Ein anderer Ansatz ist zweitens mit der offiziellen Definition kompatibel und knupft an Volksverhetzung, Rassismus und/oder Diskriminierung an. Die Vertreter_innen der ersten Perspektive kommen gleichzeitig als Produzent_innen des fraglichen sprachlichen Materials in Frage und verteidigen damit ihre eigenen Äußerungen. Eine Auswahl von entsprechenden Verteidigungsversuchen auf der Mikrobloggingplattform Twitter soll hier zunächst näher betrachtet werden.

(1) [Adressierung] »\#Hatespeech « ist Neusprech, um friedliche Kritiker v. wirklich hasserfullten Figuren wie Volker \#Beck mundtot zu machen. ): \#GKPsymp [plus Bild mit der Aufschrift]: Truth it's the new hatespeech "During times of universal deceit, telling the truth becomes a revolutionary act. " George Orwell. (2016-11)

(2) Wann wird aus Kritik Hatespeech? Komischerweise immer genau dann, wenn ihr anderer Meinung seid: ${ }^{\wedge}$ ) \#againsthatespeech (2016-11)

(3) Thr müsst alles aufsaugen und verinnerlichen, was euch der nette Onkel in den Nachrichten sagt!Widerspruch ist Hatespeech \#againsthatespeech (2016-11)

(4) Wann ist es \#hatespeech? Immer dann, wenn sich durch Deine Meinung jemand anderes angepisst fühlt. Also immer. \#againsthatespeech (2016-11)

(5) So Hashtags wie \#againsthatespeech einfach mal als Untergrabung der Meinungsfreiheit verstehen und nicht als scheinheiligen Kampf gg Hasz (2016-11)

(6) [...] Nur, dass heute Argumente als \#Hatespeech verunglimpft werden. Lachhaft fur die alte Tante SPD (2017-05)

(7) \#hatespeech ist ein Mythos, erschaffen, um das Grundrecht auf freie Meinungsăußerung einzuschränken. GEZ-Fachos, geht auf Sendepause! (2017-05)

In diesen Tweets wird Hate Speech jeweils unterschiedlich konzeptualisiert, in (1) werden Äußerungen, die mit Hate Speech etikettiert werden, 
als WAHRHEIT ${ }^{12}$ kategorisiert, in (2) als KRITIK, in (3) als WIDERSPRUCH oder auch Aufruf zu kritischer Rezeption von Nachrichten und dem daraus folgenden WIDERSPRUCH, in (4) als MEINUNGSVERSCHIEDENHEIT oder auch ZENSUR, in (5) als MEINUNGSFREIHEIT, in (6) als ARGUMENTATION und in (7) als MYTHOS. Alle diese Interpretationen laufen in etwa in der Bedeutung "unerwünschte nicht offentlichkeitstaugliche Gegenrede« zusammen. Dass Hate Speech keinesfalls als Kritik verharmlost werden kann, lässt sich klar daran festmachen, dass Kritik gănzlich ohne Verbalagressivitäten, Schuldzuweisungen, allgemeine Diskreditierungen oder Stereotypkodierungen auskommt. ${ }^{13}$

Eine neue Bedeutungskomponente wird markiert, wenn Hate Speech als NORMALFALL deklariert wird, wie in (8).

(8) Wer im Internet present ist und sich durch \#Hatespeech angegriffen fuhlt hat dort nichts zu suchen. (2017-05)

Dieser Tweet mag als Anspielung auf den raueren Umgangston im Netz ${ }^{14}$ zu verstehen sein, was jedoch gleichzeitig die Hate Speech-Problematik verharmlosen würde. Zum einen lässt sich ein etwaiger "Ton" im Netz nicht verallgemeinernd konstatieren. Neben verbaler Gewalt findet sich ein geradezu unüberschaubarer Anteil an Alltagskommunikation, die unmarkierte und auch deutlich Wertschătzung signalisierende Formen auf-

12 In der Linguistik wird diese Notation verwendet, um die Konzeptebene zu markieren.

13 Als Beispiel fur Kritik nennen Schwarz-Friesel/Reinharz, Die Sprache der Judenfeindschaft im 21. Jahrhundert, 2013, S. 261 f., den folgenden Beleg: »Es ist ganz schwer fur die Menschen hier in Deutschland, die Situation im Nahen Osten richtig ein zu schătzen, zumal die Berichterstattung einseitig ist und Dinge falsch darstellt, weil sie aus dem Kontext gerissen sind. Bitte gestatten Sie mir trotzdem die Äußerung einiger Gedanken zu obigen Konflikten. [...] Die Lebenssituation in Israel erfordert wohl besondere Maßnahmen; und ich kann möglicherweise nicht nachvollziehen, wie schwer und unsicher ein Leben in Ihrem Land ist. Trotzdem komme ich mit der Politik Israels nicht zurecht. Und ich bitte Sie von Herzen, auf Ihre Regierung ein zu wirken, daß sie andere Wege als den Krieg sucht und die Friedensgespräche fortsetzt bzw. intensiviert und Gespräche mit der Autonomiebehörde im Gazastreifen aufnimmt. Ich bedanke mich bei Ihnen, daß Sie meinen Ausfuhrungen Aufmerksamkeit geschenkt haben."

14 Siehe dazu unter anderem Feldweg/Kiebinger/Thielen, Zum Sprachgebrauch in deutschen Newsgruppen, in: Schmitz (Hrsg.), Neue Medien, Osnabrücker Beiträge zur Sprachtheorie (OBST) 1995, S. 143, und Kleinke, Sprachliche Strategien verbaler Ablehnung in offentlichen Diskussionsforen im Internet, in: Herrmann/Krămer/Kuch (Hrsg.), Verletzende Worte. Die Grammatik sprachlicher Missachtung, 2007, S. 311-336. 
weist. Zum anderen weist selbst der vielzitierte "raue Umgangston « Nuancen auf, die es zu differenzieren gilt. Ich komme darauf in Kapitel 3 dieses Artikels zurück. Dass das Konzept HATE SPEECH konsequent überstrapaziert wird, zeigt denn auch Beispiel (9) deutlich:

(9) War das von Lammert am Freitag nicht eigentlich auch \#HateSpeech!? (2017. 06)

Der Bundestagspräsident hatte gegenüber Erika Steinbach klargestellt, dass Bundestagsabgeordnete Entscheidungen unabhängig von Freigaben durch Fraktionen und Parteien treffen und Gewissensentscheidungen einzig von der Instanz »jeweiliger Bundestagsabgeordneter« abhängig seien, eine im Zusammenhang mit Hate Speech vollkommen unverdächtige ÄuBerung ${ }^{15}$ also. Selbst der deutliche Verweis vom Rednerpult, ${ }^{16}$ an das Erika Steinbach noch einmal herantreten wollte, enthält keinerlei Despektierlichkeiten, sondern spiegelt nur die Einhaltung der Regeln, die fur alle Redner_innen im Bundestag gelten. Im Diskurs wird eine große Unsicherheit darüber artikuliert, wie Hate Speech zu definieren sei, siehe auch Beispiel (10).

(10) Lasst uns \#Hatespeech sperren! Was ist das eigentlich? Wissen wir auch nicht so genau ... aber es gehort gesperrt. (2017-05)

Das wird zum Anlass genommen, mit der Bedeutung zu spielen, sie polemisch in alle Richtungen auszuweiten, und damit das dahinterstehende Konzept ad absurdum zu fuhren. Wenn hier vorgetăuscht wird, dass jede noch so harmlose Äußerung, jede berechtigte Kritik, jeder nachweisbare Fakt und die Wahrnehmung eines demokratischen Grundrechts (seine Meinung frei zu äußern) mit Hate Speech gleichzusetzen sei, wirkt das auf die Diskriminierungsdebatte zurück, die als nicht zielführend, ja diffus markiert wird.

15 Norbert Lammert am 30.6.2017 nach einem Redebeitrag von Erika Steinbach: "Nach unserer Verfassung entscheidet jeder einzelne Abgeordete, wie er sich zu jedem einzelnen - [...]- entscheidet jeder einzelne Abgeordnete, wie er sich zu welchem beliebigen Tagesordnungspunkt auf der Tagesordnung des deutschen Bundestages verhallt. Dazu bedarf es keiner Freigabe, weder durch Fraktionen noch durch Parteien. Und auch furr die viel strapazierte Frage, was denn eine Gewissensentscheidung sei, gibt es eine einzige, zustăndige Instanz: Und das ist der jeweils einzelne Abgeordnete. Es wäre schön, wenn das für die $\mathrm{Zu}$ kunft unmissverstăndlich deutlich wurde."

16 Norbert Lammert am 30.6.2017 gegenuber Erika Steinbach: »Sie haben jetzt nicht nochmal das Wort, ich hab jetzt nicht die Absicht mit Ihnen eine Debatte zu fuhren.« 
Der zweite Diskursstrang zeigt nun, dass Hate Speech mit Volksverhetzung gleichgesetzt und Hetze klar von freier Meinungsăußerung unterschieden wird:

(11) \#againsthatespeech? Aber dann darf ich ja nicht mehr als Meinung getarnte Volksverhetzung betreiben! Mimimi! (2016-11)

(12) Plumpe Hetze gegen Minderheiten, weil man was weis ich fur Probleme hat, sind keine Meinung, sondern nur Hass \#againsthatespeech (2016-11)

Die Vertreter_innen der zweiten Perspektive kommen als Adressat_innen in Frage oder als Regulierungsinstanzen. Tweets wie 11 und 12 bilden das andere Ende einer Skala, entlang derer verschiedene Auslegungen von Hate Speech anzusiedeln sind. In deren Nahe sind weite Definitionen zu verorten, wie sie u. a. von Renate Künast (und/oder ihrem Social-MediaTeam) vorgenommen werden.

\section{Beschreibungsversuche von Nutzer_innen}

So spricht sie auf ihrer offiziellen Facebookseite von Hasskommentaren, worunter aber auch persönliche Beleidigungen fallen. Unter Netiquette findet sich nämlich ein Hasstool, das folgendermaßen eingeführt wird:

Hallo,

Sie wollen mir einen Hass-Kommentar schicken? Sich mal so richtig auskotzen? Vielleicht weil ich in einer Talkshow nicht das erzăhlt habe, was Sie hören wollten? Oder weil Ihnen meine Politik nicht passt? Oder weil Sie meine Frisur nicht mögen?

Sie wissen aber noch nicht genau, was Sie schreiben sollen? Oder Sie haben eine ausgepragte Rechtschreibschwăche? Dann gebe ich Ihnen hier ein paar Hinweise, die Ihnen das Schreiben und mir das Lesen erleichtern:

Die hier genannten Aspekte spiegeln die bisherigen Erfahrungen mit hier so genannten Hasskommentaren wider, zielen aber alle darauf ab, dass die Politikerin persónlich angegriffen wird. Sie beziehen sich a) auf die "Grußformel«, die von "Frau Kunast « uber »Frau Knast« bis hin zu "Sehr geehrte Frau Kunast « changieren konnte, b) auf den Inhalt, der innerhalb des Hasstools weniger spezifiziert wird, sondern mit Zitaten illustriert wird, die expressiv-evaluierend ausfallen, wie "pfui!!!!«, wunerträglich!!!!!«, »peinlich!!!!!!«, »dumm wie Brot« oder sogar diffamierend, wie etwa »Volksverrăterin! « Unter dem Punkt Inhalt wird zudem die Dynamik von kollaborativem Hass angesprochen: 
Es gibt natürlich noch viel mehr Möglichkeiten. Schauen Sie sich die Kommentare Ihrer Vorgängerinnen und Vorgänger an. Kopieren Sie es einfach. Hauptsache, es geht Ihnen danach besser.

Ein dritter Aspekt (c) wird hier mit "Stil« überschrieben und bezieht Beobachtungen zur exzessiven Verwendung von Ausrufezeichen und Marginalien ein. Unter dem Punkt "Soziales" (d) werden die positiven Rückmeldungen anderer Nutzer_innen thematisiert, die bei gegenseitigem $\mathrm{Li}$ ken zu einer »Gruppenerfahrung [...] « beitragen können. Unter dem letzten Punkt (e) werden die "Konsequenzen« für das Versenden von Hasskommentaren konkretisiert, sie bestehen in "Strafanzeigen wegen Beleidigung und Volksverhetzung ".

Hier wird also letztlich völlig richtig zwischen Beleidigung und Volksverhetzung unterschieden. Beispiele werden jedoch nur für Beleidigungen gegeben. Beleidigungen sind daran zu erkennen, dass sie im Unterschied zu Hassrede dann vorliegen, wenn eine Person wals Individuum verunglimpft oder herabgewürdigt wird, also nicht als Mitglied einer Gruppe oder über seine Zugehörigkeit zu dieser Gruppe. ${ }^{17}$ Im vorliegenden Fall wird beides als "Hasskommentar« etikettiert. Die aufgeführten Charakteristika a) bis e) sind zwar Anhaltspunkte, aber keine Kriterien der Ausschließlichkeit. Es zeigt sich also auch hier, dass der Terminus Hassrede/Hasskommentar sehr weit und unspezifisch verwendet wird, es aber doch Bemühungen gibt, die Bedeutung einzugrenzen.

In einem Spot der Tagesschau wurde beispielsweise versucht, das Phänomen Hate Speech möglichst kompakt zu definieren (siehe Abb. 1): ${ }^{18}$

17 Stefanowitsch (Fn. 11).

18 Eine dritte Gruppe von Personen, die das Netzwerkdurchsetzungsgesetz kritisieren, dabei aber Hate Speech nicht in Frage stellen, wird hier nicht weiter berücksichtigt. 


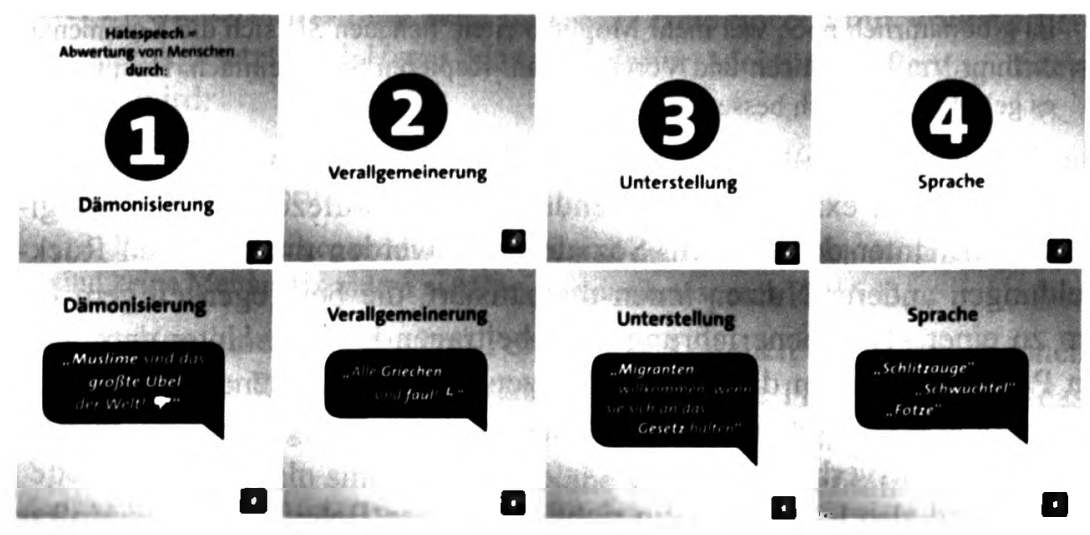

Abb. 1: Bildschirmfotos aus dem Tagesschau-Video zum Thema "Wie erkenne ich hatespeech? « ${ }^{19}$

Hate Speech wird als "Abwertung von Menschen « wegen Herkunft, Religion, Hautfarbe, Sexualität und Geschlecht deklariert. Als Abwertungsstrategien werden Dämonisierung, Verallgemeinerung und Unterstellung genannt, als vierter Faktor erscheint Sprache. Jede dieser Kategorien wurde mit einem Beispiel unterlegt, drei davon stammen aus einem Aufsatz von Anatol Stefanowitsch. In "Was ist überhaupt Hate Speech? « fasst der Sprachwissenschaftler die wichtigsten Kriterien zur Abgrenzung präzise zusammen. ${ }^{20}$ Die genauen Entstehungsbedingungen für das Video sind mir nicht bekannt, so weiß ich nicht, inwieweit die Tagesschau linguistisch fundierte Beratung über die Rezeption des Artikels von Stefanowitsch hinaus in Anspruch genommen hat, es scheint mir aber so, als wäre hier auf (seine) weitere fachliche Unterstützung verzichtet worden.

Von dem Versuch, eine solche komplexe Thematik in einem 56sekündigen Video erklären zu wollen, hätten Expert_innen dem Team der Tagesschau vermutlich abgeraten. In dieser kurzen Zeit bleibt nur wenig Raum für Erklärungen, die gerade aber bei sprachlichen Äußerungen so wichtig sind, weil sich Bedeutung immer erst im konkreten Kontext ausdifferenzieren lässt. Letztlich hat sich das Video insofern als kontraproduktiv erwiesen, als dass es emeut Hate Speech evoziert hat, siehe 13 und 14.

19 https:/www.tagesschau.de/multimedia/video/video-293651.html.

20 Stefanowitsch (Fn. 11). 
(13) Die Griechen sind doch nicht faul! Sie sind den ganze Tag damit beschäftigt ihr Hab und Gut vor den Steuern zu versteckenitro (2017-05)

(14) Warum sollen Griechen denn fleissig sein bekommen doch immer Hilfspaket, (2017-05)

Auf der Seite unser-mitteleuropa.com heißt es:

Besonders der dritte Punkt macht stutzig. Zu »Hate Speech “ wird ein Kommentar erklärt, der wortwörtlich folgenden Inhalt hat: "Migranten willkommen, wenn sie sich an das Gesetz halten «. Was daran falsch sein soll, fragt sich vermutlich jeder, der das Video gesehen hat. $\mathrm{Zu}$ fordern, dass sich die abendlandunterwandernden Invasoren wenigstens an deutsches Recht halten müssen, ist nun also auch schon "Hate Speech $\ll$.

In einem Facebook-Eintrag von "Lügenpresse « wird der Satz als "harmlos« bezeichnet, im folgenden Tweet wird die Brisanz der Aussage ignoriert:

(15) Wenn das Formulieren von Bedingungen für die Einwanderung als Hatespeech ausgelegt werden kann, dann taugt die Definition nichts. (2017-05)

\section{Einbettung in linguistische Ansätze}

Es handelt sich hier keinesfalls um einen harmlosen Satz, denn er spiegelt das Vorurteil, Migrant_innen hielten sich per se nicht an Gesetze. Die Rekonstruktion solcher Vorurteile gelingt unter Rückgriff auf lange etablierte pragmalinguistische Theorien, wie etwa die Implikaturentheorie. ${ }^{21}$ Dieser Theorie liegt die Annahme zugrunde, dass Menschen sich in Kommunikationssituationen grundsätzlich kooperativ verhalten. Dieses kooperative Verhalten zeichnet sich durch die Einhaltung von vier Maximen aus:

1. Maxime der Quantität: Mache deinen Beitrag so informativ wie (für die gegebenen Gesprächszwecke) nötig und mache deinen Beitrag nicht informativer als nötig.

2. Maxime der Qualität: Versuche deinen Beitrag so zu machen, dass er wahr ist. Sage nichts, was du für falsch hältst. Sage nichts, wofür dir angemessene Gründe fehlen.

3. Maxime der Relation: Sei relevant.

21 Grice, Logik und Konversation, in: Meggle (Hrsg.), Handlung, Kommunikation, Bedeutung, 1979, S. 243-265. 
4. Maxime der Modalität: Sei klar. Vermeide Dunkelheit des Ausdrucks. Vermeide Mehrdeutigkeit. Sei kurz (vermeide Weitschweifigkeit). Der Reihe nach! $!^{22}$

Die Maximen sind nicht normativ zu verstehen, sondem als Erwartungen, die an jede Konversation gestellt werden. Ein Verstoß gegen eine oder mehrere dieser Maximen ist kein Regelbruch, sondern er kann als Einladung zur Umdeutung einer Äußerung verstanden werden. Im vorliegenden Beispiel wird offensichtlich gegen die Maxime der Quantität verstoßen, weil für die Botschaft, dass "Migranten willkommen« sind, mehr Informationen als notwendig gegeben werden.

Der Zusatz "wenn sie sich an das Gesetz halten « wird hier als notwendige Bedingung für das Willkommensein expliziert. Das ist deshalb markiert, weil grundsätzlich davon auszugehen ist, dass sich alle Menschen an das Gesetz halten, also auch Migrant_innen. Ein solcher Zusatz ist folglich redundant, es sei denn, man möchte damit eine weitere Aussage, eine lmplikatur verknüpfen. Implikaturen sind als Bedeutungen zu verstehen, die über das wortwörtlich Gesagte hinausgehen. Die Schlussfolgerungen, die von Rezipient_innen gezogen werden, können dann jeweils negiert werden. In diesem Fall handelt es sich bei einer solchen Schlussfolgerung darum, dass unterstellt werden soll, Migrant_innen hielten sich nicht an Gesetze. Eine Rücknahme/Negation dieser Bedeutungsrekonstruktion könnte dann etwa lauten: Migranten willkommen, wenn sie sich an das Gesetz halten. Damit will ich aber nicht sagen, dass sich Migranten sonst nicht an Gesetze halten. Es sei aber hier eingeräumt, dass die Konditionalsatzeinleitung wenn die Rücknahme einer solchen Deutung schwer nachvollziehbar erscheinen lässt.

Wer einen solchen Satz nun als "harmlos « einstuft - wie im erwähnten Facebook-Kommentar - geschehen, zeigt damit an, dass er/sie das Vorurteil bereits internalisiert hat und/oder eine Legitimation für dessen Verbreitung voraussetzt. Es zeigt sich $\mathbf{u}$. a. an diesem Beispiel, dass wir über verbale Äußerungen "Aufschluss über Einstellungen [und] Weltbilder einzelner Sprachbenutzer $\left[\ldots . . .{ }^{23}\right.$ erhalten. Aus diesem Grund ist Hate Speech

22 Grice (Fn. 21), S. 243-265.

23 Schwarz-Friesel, Spannung in Texten erklaren: eine kognitions- und korpuslinguistische Analyse im Rahmen der Textweltmodelltheorie, in: Marx/Meier (Hrsg,), Sprachliches Handeln und Kognition - Empirische Analysen, 2018. 
eine aufschlussreiche Datengrundlage, wenn es darum geht Diskriminierungstendenzen in einer Gesellschaft zu eruieren.

Ein weiterer Kritikpunkt am oben vorgestellten Versuch, Hate Speech zu beschreiben, setzt am vierten Kriterium an. Abgesehen davon, dass die Bezeichnung Sprache sehr unglücklich gewählt ist, weil natürlich auch die genannten Strategien Dämonisierung, Verallgemeinerung und Unterstellung sprachlich umgesetzt werden, ist es problematisch, vom Kontext losgelöste Schimpfwörter zu zitieren, die Hate Speech indizieren sollen. Es wird hier überhaupt nicht in Zweifel gezogen, dass Hate Speech häufig mit der Verwendung pejorativ konnotierter Wörter einhergeht. Konnotationen sind intersubjektive emotive Nebenbedeutungen eines Wortes. ${ }^{24}$ Die Verwendung konnotierter Wörter ist dann offensichtlich, wenn neutrale oder meliorative Alternativen zur Referenzialisierung zur Verfügung stünden, etwa wenn ein Obdachloser als Penner bezeichnet wird. Pejoration ist auch auf der Wortbildungsebene erkennbar, so wirkt sich z. B. die Endsilber -ler abwertend aus. ${ }^{25}$ Es ist jedoch wichtig, dass von der Verwendung von Schimpfwörtern oder pejorativ konnotierten Bezeichnungen nicht automatisch rückgeschlossen wird, dass es sich um Hate Speech handelt. Beim sogenannten Bantern ${ }^{26}$ (oder auch Dissen) ${ }^{27}$ dienen degradierende Bezeichnungen der gruppeninternen Hierarchisierung und Ratifizierung als Gruppenmitglied, sie werden gleichzeitig scherzhaft verhandelt. Stefanowitsch führt ein weiteres Beispiel an, das zeigt, wie schwierig der Rückschluss von einzelnen Wörtern auf Hate Speech ist: So richtet sich ein Wort wie Schwuchtel als Hassrede gegen die so bezeichnete Gruppe (homosexuelle Männer). Wird das Wort jedoch gegenüber einer Person geäußert, die nicht zu dieser Gruppe gehört, wird diese Person "nur« beleidigt. ${ }^{28}$

24 Vgl. Schwarz/Chur, Semantik: Ein Arbeitsbuch, 2014, S. 234.

25 Vgl. Stefanowitsch (Fn. 11).

26 Marx, «Kaum [...] da, werd' ich gedisst! « Funktionale Aspekte des BanterPrinzips auf dem Online-Prufstand, in: Spiegel/Gysin (Hrsg), Jugendsprache in schulischen, medialen und offentlichen Răumen, 2016, S. 287-300.

27 Dissen wird von Deppermann/Schmidt als maggressives Sprechen « beschrieben, das eine "Doppelstruktur von Spaß und Emst《 aufweist, s. Deppermann/ Schmidt, Dissen: Eine interaktive Praktik zur Verhandlung von Charakter und Status in Peer-Groups männlicher Jugendlicher, Osnabrücker Beitrăge zur Sprachtheorie (OBST) 2001, 79. Stefanowitsch (Fn. 11). 


\section{Hate Speech: Ausdruck von Emotionen oder Weltbildern?}

Sprache fordert Denkschemata zutage, das wurde oben gezeigt. Entsprechend drastische Sprache lässt im Zusammenhang mit Hate Speech auf eine Verrohung einzelner Sprachverwender_innen schließen oder darauf, dass dieser Eindruck erweckt werden und strategisch eingesetzt werden soll, um beispielsweise Unruhe zu stiften oder Gesinnungskoalitionen im Diskurs zu bilden. Das bedeutet also auch, dass Hassrede nicht automatisch ein Ausdruck von tief empfundenem Hass ist, die Hassredner_innen müssen Hass nicht subjektiv empfinden. Vielmehr kann Hassrede konventionalisiert sein, wie Van Dijk (2003) feststellt. ${ }^{29}$ Das ist teilweise im rassistischen Diskurs der Fall. "Hass«, so fasst es auch die Autorin Carolin Emcke "ist kollektiv und er ist ideologisch geformt. " $^{30}$

Aus psychologischer/neurobiologischer Sicht ${ }^{31}$ ist Hass eine Strategie zur Bewältigung von Angst. Kemberg zufolge leitet sich Hass aus Wut $a b^{32}$ Diese Wut kann verschiedene Ursachen haben und durchaus eine Reaktion auf gefuhlte Ohnmacht in Angstsituationen sein. Sie gilt als "Primäraffekt, der zum Kristallisationspunkt für den Aggressionstrieb wird ${ }^{33}$. Hass zeichnet sich dadurch aus, dass Menschen gehassten Personen keine Empathie entgegenbringen und ihre Hemmungen ihnen gegenuber verlieren. ${ }^{34}$ In einem kürzlich erschienenen Zeitungsartikel spricht die Antisemitismusforscherin Monika Schwarz-Friesel von Hasssprache als geistiger Gewaltanwendung. ${ }^{35}$ Aggressionen können sowohl auf lexikalischer Ebene transportiert werden, etwa durch die Verwendung pejorativer Lexeme in entsprechendem Kontext, durch Intensivierer oder skan-

29 Van Dijk, Racist Discourse, in: Cashmore (Hrsg.), Routledge Encyclopedia of Race and Ethnic Studies, 2003, S. 351-355.

30 Emcke, Gegen den Hass, 2016.

31 Vgl. Hüther im Gesprăch mit Kropnik, 2016, http://wiener-online.at/ 2016/09/21/interview-mit-gerald-huether/, abgerufen am 25.7.2017.

32 Kemberg, Wut und Hass: über die Bedeutung von Aggression bei Persönlichkeitsstörungen, 3. Aufl. 2003, S. 36.

33 Kemberg (Fn. 32), S. 35.

34 Rosenbrock, Die antifeministische Männerrechtsbewegung. Denkweisen, Netzwerke und Online-Mobilisierung. Eine Expertise für die Heinrich-BöllStiftung, in: Heinrich-Bbll-Stiftung (Hrsg.), Schriften des Gunda-WernerInstituts, 2016, S. 16, https:/www.boell.de/de/content/die-antifeministischemaennerrechtsbewegung, abgerufen am 25.7.2017.

35 http://www.juedische-allgemeine.de/article/view/id/29101. 
dalisierenden Wortschatz. ${ }^{36}$ Negativ-Emotionen lassen sich aber auch auf syntaktischer Ebene ablesen, an Mustern wie Substantiv plus Richtungsangabe (Ausländer raus!) ${ }^{37}$ z. B., an emphatischen Ellipsen (Kanake!) oder auch an der Anhäufung von Satzendzeichen, insbesondere Ausrufezeichen.

Inhaltlich finden wir wiederkehrende Themen, wie Altparteien, Lügenpresse, Flüchtlingsproblematik, Forderung nach mehr plebiszitären Elementen $^{38}$, deren Darlegung und Diskussion einseitig verläuft und eigene Eindrucke und Empfindungen (die an ideologisch Gleichgesinnten gespiegelt werden) über Fakten und/oder Expert_innenwissen stellt.

Es handelt sich hier um eine Überkompensation zutiefster Verunsicherung, die sich jedoch verselbstständigt hat. So wird der Hate SpeechDiskurs nicht vordergrilndig von Angst und Verunsicherung motiviert, sondern von den diese Emotionen begleitenden Schutzmechanismen der Abgrenzung, die sich verfestigt haben, denn sim Gegensatz zur Heftigkeit von Wutreaktionen und zur Wechselhaftigkeit der kognitiven Aspekte von Ärger und Wut ist der kognitive Aspekt von $\mathrm{Haß}$ dauerhaft und beständig. 13

\section{HateSpeech als Marker für Abgrenzung: Explikation am Beispiel}

Damit einher gehen ganz konkrete Zuschreibungen wie "wir« gegen »die«, was zur Bildung einer kollektiven Identităt beiträgt. ${ }^{40} \mathrm{Im}$ folgenden Beispiel, einem an die Journalistin Dunja Hayali persönlich gerichteten Hassbrief, lässt sich das nachzeichnen:

(16) Frau Hayali, Sie sind eine der dümmsten Ziegen, die beim ZDF arbeiten Wenn ich morgens meinen Fernseher anschalte und Dich arabisches Stückchen Scheisse sehe, ist mir der ganze Tag versaut. Und nicht mal die Frau Petra, die ja so viele Stimmen im ganzen Land bekam, mochte sich mit so einer Eselvickerin wie Sie, abgeben. Ich brauche als gesetztestreuer hochqualifizierten Bürger mit Ihre Einlassungen und dummes Gesabbert nicht ansehen. Und dann ihr Aussage sie Hơhlenscheisserin, die sich früher mit einer Hand voll Wüstensand, gemischt mit

36 Vgl. Scharloth, Ist die AfD eine populistische Partei? Eine Analyse am Beispiel des Landesverbandes Rheinland-Pfalz, Aptum 2017, S. 1-15.

37 Vgl. Stefanowitsch (Fn. 6).

38 Scharloth (Fn. 36).

$39 \quad$ Kemberg (Fn. 32), S. 37.

40 Rosenbrock (Fn. 34), S. 16. 
Eselshaare den dreckigen Arsch abgewischt hat, dass sie sich mit Pfefferspray bewaffinet habe, , ist doch lächerlich. Pfefferspray gegen Menschen ist doch verboten, natürlich darfst du Dreckstuck das jederzeit, mit der Bewilligung deines Kalifen einsetzen. Wer dich die im Dunkeln mitnimmt und am Tage bei Licht ansieht, bringt dich unbescholten wieder und ist froh, dass er dich los ist.

Ich hoffe es gibt eines Tages eine Volksbefragung und wir können das ganze faule Ausländerpack wie dich los werden. Du wirst dann Klofrau auf dem Hauptbahnhof in Bagdad.. Denn du sind doch eine der größten Strolche uberhaupt. Du hast mit durchgesetzt, dass jeder Haushalt Fernsehgebuhren zahlen muss, auch die Leute die ein sehr geringes Einkommen haben. Mit deren Geld kannst du dann protzen, dir selbst und deiner Bagage dumme Auszeichnungen umhăngen und immer mehr ausländisches Pack beschaftigen. [...] Also verpiss dich aus dem ZDF und bitte um Asyl in Indien, da gibt es noch Männer die eine wie dich vŏgeln ! PS: Dieses Schreiben habe ich auch schon an die Polizei, die Auslănderbehörde. T-Online, die BAMF und an Herm Bellut geschickt, das brauchst du dann nicht mehr machen. Und hole dir wie Frau Merkel einen neuen Personalausweis . so wie der beiliegende [Abbildung einer Ausweiskopie mit verfalschtem Foto und Namen von Angela Merkel]. Gruß Lawrence von Arabien ${ }^{41}$

Der/die Schreiber_in des Briefes generiert hier klare Kontrapositionen, in dem die Adressatin des Briefes als die wandere « inszeniert wird:

a) durch Dehumanisierungen (Ziege, Stückchen Scheisse, Dreckstück, Sau $^{42}$, Ferkel $^{22}$, Schwein $^{22}$ ) als nicht-menschlich,

b) durch regionale Zuschreibungen (arabisch, Wüstensand, Ausländerpack, Bagdad, ausländisches Pack, Arabien, Asyl in Indien, Ausländerbehörde) als nicht-deutsch,

c) durch Religionszugehörigkeitszuschreibungen (Kalif) als nicht-christlich,

d) durch so bezeichnete Straftaten (Pfefferspray gegen Menschen ist doch verboten, Mit deren Geld kannst du dann protzen, Polizei) als nichtgesetzestreu

e) durch Referenzen auf Körperhygiene (dreckigen Arsch, benutzte Toilettenpapier und wasche gefälligst [...] deine unreine Hand ${ }^{22}$, Deine Fingernägel färbst du ja immer schon rot [...] weil man ansonsten die Scheissenreste darunter sieht ${ }^{22}$, Spinnt aus dem es ganz erbärmlich stinkt ${ }^{22}$ ) als unsauber.

41 Es handelt sich hier um einen zwei DIN-A4-Seiten umfassenden Brief, den die Journalistin uber ihre Social Media-Accounts zugänglich gemacht hat. Fettdruck aus dem Original lubernommen.

42 Im hier nicht zitierten Teil des Hassbriefes. 
Diese Eigenschaften sollen jeweils im Gegensatz zum/zur Schreiber_in des Briefes gelesen werden, auf den/die diese nicht zutreffen. Darüber hinaus beschreibt er/sie sich selbst als gesetztestreue[n], hochqualifizierten Bürger.

Auffallig sind degradierende und die Wirkung verstärkende Attribute wie faules in faules Ausländerpack, dreckigen in dreckigen Arsch oder dümmste in dümmste Ziege. Die beschriebenen Szenarien [Arsch $a b[\ldots]$ wische[n] mit Wüstensand gemischt mit Eselshaare[n], Klofrau auf dem Hauptbahnhof von Bagdad als Resultat einer Volksbefragung, Bitte den Bellt um die Auslage einer Fingernagelbürste auf deiner Damentoilette. Deine Fingernägel färbst du ja schon immer rot, wie man sich unter den Damen erzählt. ${ }^{22}$ Onanie mit mit Eselsperma eingeriebenen Rüben in Arabien] sind gekennzeichnet von einer Akkumulation an vollkommen absurden Stereotypkodierungen. Ereignisse, die stattgefunden haben, etwa die Auszeichnungen, die die Journalistin erhalten hat, werden in diese Szenarien integriert, was denn auch zu deren Abwertung beiträgt.

Hier wird ein Versuch unternommen, eine Persönlichkeit zu dekonstruieren. Die Adressatin wird nicht nur eklatant in ihrer Integrität verletzt, sondern auch durch die Angriffskaskaden bedroht. Es ist kaum abzuschätzen, ob die hier versprachlichte Aggression nicht auch in tätliche Gewalt umschlagen kann. Das Potenzial ist zweifelsohne in diesem Brief wie in Hassrede generell angelegt. ${ }^{43}$

Durch eine Reihe von Inkohärenzen (u. a. der Name, den sich der/die Schreiber_in/nen selbst geben: Lawrence von Arabien, mit dem eine $\mathrm{Zu}$ gehörigkeit zu einer Gruppe, die im Hassbrief attackiert und degradiert wird, impliziert wird) und Wiederholungen (etwa das Höhlen-Thema), aber auch durch die Drastik der genannten Szenarien entsteht der Eindruck, der Hassbrief sei das Ergebnis eines kollektiven Schreibprozesses: Hier scheinen sich mehrere Ideengeber_innen überbieten zu wollen. Dieser Eindruck wird dadurch gestützt, dass innerhalb des Hassbriefes zwi-

43 Vgl. dazu Herrmann/Kuch, die das Verhältnis zwischen Sprache und Gewalt in die drei Ansătze fassen: Gewalt und Sprache (1), Gewalt der Sprache (2) und Gewalt durch Sprache (3). Unter (1) fällt das temporäre Verhältnis von Gewalt als vorgăngig oder nachträglich. Sprache wird hier also als ein Mittel betrachtet, über das physische Gewalt angekündigt werden kann (etwa durch Drohungen), und als Beschreibungs- und Kategorisierungsinstrument in der Reflexion physischer Gewalt: Herrmann/Kuch, Verletzende Worte. Eine Einleitung, in: Herrmann/Krămer/Kuch (Hrsg.), Verletzende Worte. Die Grammatik sprachlicher Missachtung, 2007, S. 7-30. 
schen der Distanzlosigkeit signalisierenden und Beschimpfungen einleitenden Du-Anrede und dem Sie gewechselt wird, das möglicherweise zum Anschein beitragen soll, es handle sich um einen formellen Beschwerdebrief (Sie sind eine der [...] vs. Dich arabisches [...]; so einer [...] wie Sie, Ihre Einlassungen, ihre Aussage, sie Höhlenscheisserin, [...] dass sie sich mit Pfefferspray [...] vs. darfst du Dreckstück; wer dich, bringt dich [...] wieder, dass er dich los ist; Unter ihrer Mitarbeit vs. mit wem du Sau usw.).

Da es sich hier um einen ausgedruckten Brief handelt, der der Adressatin zugesendet wurde, kann die Vermutung, dass er von mehreren Autor_innen erstellt wurde, nicht verifiziert werden. Generell sind solche Dynamiken im Zusammenhang mit Hate Speech nicht ungewöhnlich. Am folgenden Beispiel kann man gut nachvollziehen, wie sich die einzelnen Beiträger_innen gegenseitig bestärken und zu übertreffen versuchen.

\section{Hate Speech als Produkt kollaborativer Dynamik}

Beispiel (17) stellt einen Ausschnitt der Reaktionen auf eine FacebookStatusmeldung von Lutz Bachmann 3 dar, in der er als Thema die "Geisteskranke " (unter Bezugnahme auf eine Aussage des amerikanischen Präsidenten Donald Trump), IMErika, Kanzlerin derSchande und Volksverräter installiert und damit auf die Bundeskanzlerin Angela Merkel Bezug nimmt.

A Die Merkel soll sich doch nach Afrika scheren und da unten kandidieren, dann kann sie soviel Flüchtlinge holen wie sie will [...]

B Eines Tages scheißt Dein Volk Dir vor Deine Tur. Und verbietet Dir Deine DDR Taucherbrille!

C Anstatt den Menschen vor Ort in ihrer Heimat zu helfen quatscht die von legaler Migration damit keine mehr ertrinken das ist total verrilukt und pervers

D Diese verlogene, verkommene alte Hexe.

E Diese Frau soll ihr Lügenmaul halten

F daran ersticken soll Sie

G Wenn ich den Schleusern entgegenwirken will, kann ich das schon beim Besteigen der Boote tun! [...]

H vielleicht sollte sie lieber ganz vorsichtig sein... und niemand reglementieren, der ihr mal die wahrheit sagt. 
I Es wird doch in diesen Ländern, massiv Werbung durch die Botschaften gemacht ! Sollen alle herkommen, hier ist es so friedlich und ruhig !

J Welche Hornochsen haben dort geklatscht?

K Kann sich nicht einer Opfern der schlimm krank ist und bringt diese Person um die Ecke !?

L Insane woman, not going to win!!!

Die Kommentator_innen greifen die Namensgebung auf und erweitern das Repertoire despektierlicher Titel, indem sie auf Angela Merkel mit Die Merkel, die, diese verlogene, verkommene alte Hexe, diese Frau, diese Person und Insane woman referieren. Der Akt der Namensgebung wird mit Machtausübung verbunden. Interessanterweise fallen in der Bedeutung von heißen die Bedeutungen bezeichnen oder benennen (Ich heiße $X Y Z$, ich heiße dich YXZ) zusammen mit befehlen oder auffordern (Ich heiße dich $x y z z u$ tun). ${ }^{44}$ Durch Namensgebung wird eine Person entindividualisiert, ${ }^{45}$ indem sie etwa auf die von anderen für sie ausgewăhlte Bezeichnung reduziert und objektiviert wird, denn waller Hass von Menschen [ist] darauf aus [...], anderen Menschen ihren Status als Subjekt zu nehmen. $\ll^{46}$

Im Fall von Hate Speech geschieht das durch Negativ-Zuschreibungen. Diese entstammen nicht zufalligerweise dem semantischen Feld der Luge/Unwahrheit. ${ }^{47}$ Eine Hexe ist eine vertrauensunwürdige Märchengestalt, es ist von Lügenmaul die Rede, von Geistesgestört[heit] und vom InsaneSein - beides sind keine Indikatoren für Glaubwürdigkeit und schließlich ruft der Halbsatz mal die wahrheit sagt ein Topik auf, das sich durch den rechtspopulistischen Diskurs zieht und hier bereits angeklungen ist als die verschiedenen ethnokategorischen Auslegungen des Hate Speech-Begriffs besprochen wurden (Beispiele 1 bis 7). Damit einher geht die Annahme, die Regierung unterbinde die freie Meinungsäußerung durch eine Zensur, vgl. stellvertretend dafur den folgenden Tweet:

44 Bergmann, Die Macht des Wortes, in: Buchheim/Cierpka (Hrsg.), Macht und Abhăngigkeit, 2000, S. 120-131.

45 Butler, Hass spricht, 2006.

46 Haubl/Caysa, Hass und Gewaltbereitschaft, 2007, S. 9

47 Vergleichbare Prozesse finden wir auch in Cybermobbing-Prozessen, vgl. Marx, Diskursphänomen Cybermobbing. Ein internetlinguistischer Zugang zu [digitaler] Gewalt, 2017. 
Die ganze Diskussion ist reine Gesinnungsschnuffelei. Und ich zahl auch noch GEZ für so einen Denunziantenstadl.

Leitsprüche wie Mut zur Wahrheit und Phrasen, wie Das wird man doch wohl noch sagen dürfen implikatieren diese Annahme und damit jeweils auch die In-Frage-Stellung eines demokratischen Systems. Daraus ergibt sich der vermeintlich logische Schluss, die Demokratie existiere nicht, könne also auch gar nicht gefährdet werden. Das eigene undemokratische Handeln kann damit legitimiert werden, was mit einer Gleichsetzung eines normalen Vorgehens zusammenfallt. Diskursiv wird also ein neutraler (und nicht mehr rechtspopulistischer) Status kreiert, von dem aus weiter agiert werden kann. Das gelingt durch gegenseitige Bestärkung, wie wir sie im Beispiel (17) nachvollziehen können. Die einzelnen Kommentator_innen pflichten sich gegenseitig bei und elaborieren das Thema kontinuierlich. Vergleichbare Beispiele finden sich häufig, sie können eine Erklärung dafür sein, dass ostentative Vorurteile (wie im Hate SpeechBeispiel Migranten Willkommen, wenn sie sich an das Gesetz halten) nicht als solche dechiffriert oder schlicht negiert werden.

Über die Diskreditierung der Bundeskanzlerin hinaus kommt es in kürzester Zeit zu Gewaltphantasien bis hin zu Gewaltaufrufen. So wird mit daran ersticken soll Sie eine Verwünschung realisiert, mit vielleicht sollte sie lieber ganz vorsichtig sein...eine Drohung ausgesprochen und mit Kann sich nicht einer Opfern der schlimm krank ist und bringt diese Person um die Ecke !? eine Tötungsabsicht formuliert.

Es gibt innerhalb der Sequenz keinerlei Gegenrede, die zur DeEskalation beitragen könnte. Relativierungen, die die Basis solcher Äußerungssequenzen destabilisieren könnten, bleiben ebenfalls aus oder werden aktiv ausgeschlossen, wie Beispiel (19) zeigt. Es handelt sich hierbei auch um eine Reaktion auf die Statusmeldung von Lutz Bachmann 3, die zu Beginn des Verlaufs gepostet wurde.

A In welchen Geschichtsbüchem ist sie schon fest als »Kanzlerin der Schande« verwurzelt? Bissl viel Pathos für meinen Geschmack.

B Ich beobachte dich und deine Kom. jetzt schon ein paar Tage, deine FB Seite erweckt zwar den Anschein als wärst du »einer von uns«, deine Kom. zeugen jedoch eher vom Gegenteil!. Was bezweckst du eigentlich mit diesem Scheiß? 
C Ob solchen Gestalten Gott wirklich hold ist? $?^{48}$

Kommentator B mahnt an, dass auf der Facebook-Seite klar Position für die vorherrschende Meinung zu beziehen ist. Das Zensur-vorbereitende Vorgehen (über längere Zeit beobachten und die Präsenz der Person im Netz prüfen) wird dabei gar nicht als solches identifiziert, obgleich es in Bezug auf den Hate Speech-Vorwurf ein gängiges Muster der Gegenargumentation ist.

Dass hier symbolisch ein Maulkorb verhängt wird, wird nicht hinterfragt, vgl. C, dem/der B beipflichtet, indem er/sie A despektierlich als solche Gestalt bezeichnet. Stattdessen wird die Gruppe "uns" als Orientierungsinstanz absolut gesetzt, deren Handlungen - anders als die von A sogar vor Gott zu rechtfertigen sind, ein Umkehrschluss, der sich aus Cs Kommentar ergibt. Es zeigt sich hier exemplarisch, wie die so energisch eingeforderte Meinungsfreiheit von ihren Verfechter_innen selbst unterlaufen wird. Hier scheint Irrationalität auf, die auch im Hassbrief an die Journalistin Dunja Hayali deutlich wurde. Es handelt sich hierbei um ein wesentliches Merkmal von Hate Speech.

\section{Abschließende Bestandsaufnahme}

Als typisch für Hate Speech wurden hier die folgenden Punkte herausgearbeitet: Hate Speech ist ein Gegenstand, der einen interdisziplinären $\mathrm{Zu}$ gang verlangt. Aus linguistischer Perspektive ist besonders interessant, wie Hate Speech sprachlich umgesetzt wird und wie das Thema im Diskurs verhandelt wird. Es zeigte sich, dass wir grob von zwei Paralleldiskursen ausgehen können. Erstens gibt es einen rechtspopulistischen Diskurs, in dem Hate Speech verharmlost wird und die Reglementierung als Angriff auf die Meinungsfreiheit deklariert wird. Es gibt zweitens einen aufklärerischen Diskurs, der auf einer Metaebene geführt wird und Schutzmechanismen für diskriminierte Gruppen hervorbringen soll. An konkreten Beispielen wurde nachvollzogen, wie Hass transportiert und kollaborativ genährt wird. Dabei wurde deutlich, dass Irrationalităt und Aggressivităt bis hin zu konkreten Gewaltphantasien ineinandergreifen. Dieser Kombination ist zweifelsohne ein Bedrohungspotenzial inhärent. 